\title{
Masa pulmonar y adenopatías en paciente fumador
}

\section{Pulmonary mass and lymphadenopathies in a smoker patient}

\author{
Rebeca Longueira, Bernardo Sopeña, José Luis García-Tejedor, José Luis Lamas \\ Servicio de Medicina Interna. Hospital Xeral. Complexo Hospitalario Universitario de Vigo (CHUVI). SERGAS. Vigo
}

\section{Introducción}

La legionelosis es una enfermedad neumónica aguda causada por un bacilo gramnegativo del género Legionella, el más habitual de los cuales es Legionella pneumophila. Sus manifestaciones clínicas inespecíficas y los hallazgos radiográficos, clásicamente descritos como infiltrados pulmonares pero también variables, dificultan su diagnóstico. Describimos a continuación un caso con presentación clínica y radiológica inusual, simulando una neoplasia pulmonar.

\section{Caso clínico}

Varón de 63 años, fumador importante sin otros antecedentes de interés, que consultó por síndrome constitucional de 2 meses de evolución con pérdida de 15 kilogramos de peso. En los últimos días presentó fiebre alta y polidipsia. A su llegada a Urgencias el paciente estaba febril, taquipneico, con saturación basal de 02 del 82\% y crepitantes gruesos en el hemitórax izquierdo. Analíticamente destacaban los siguientes valores: leucocitos 20.610 células/ul (normal: 3.500-10.800) con 94\% polimorfonucleares, glucosa $527 \mathrm{mg} / \mathrm{dl}$ (normal: 73-100), sodio 153 meq/L (normal: 135-145), GOT 223 UI/L (normal: 4-37), GPT 124 UI/L (normal: 4-38), CPK 3.427 UI/L (normal: 0-170). La radiografía de tórax mostró una masa parahiliar izquierda (figura 1). La tomografía computarizada (TC) torácica de alta resolución con angio-TC realizada 24 horas después, objetivó una masa adenopática en esa misma localización, que englobaba y desplazaba la arteria y bronquio lobar inferior, con adenopatías subcarinales y pretraqueales izquierdas de tamaño valorable, y consolidación lóbulo inferior izquierdo asociada (figura 2). La sospecha inicial principal fue de carcinoma pulmonar con afectación ganglionar y neumonitis postobstructiva asociada, aunque se procedió a un exhaustivo estudio para excluir también otras patologías. Se realizó una broncoscopia diagnóstica en la que no se observaron lesiones endobronquiales,, con recogida de muestras para determinación de Mycobacterium tuberculosis, cultivo ordinario y Lowestein, siendo todos estos estudios negativos. El lavado broncoalveolar y la biopsia transbronquial no mostraron alteraciones, y la punción transtraqueal de las adenopatías mostró únicamente cambios reactivos, sin evidencia de granulomas. Desde su ingreso el paciente fue tratado con ceftriaxona a dosis de $2 \mathrm{gr} / d i ́ a$ y azitromicina $500 \mathrm{mg} / \mathrm{dí}$, con lo cual evolucionó de forma excelente, con resolución clínica y radiológica de las alteraciones descritas en los estudios previos. Las serologías para Mycoplasma pneumoniae, Chlamídia pneumoniae y Coxiella burnetti fueron negativas, pero la IgM para Legionella pneumophila resultó positiva, con una IgG negativa. El antígeno de Legionella en orina también resultó positivo. Tras 5 meses de seguimiento, el paciente continua asintomático.

\section{Discusión}

La Legionella es un bacilo gramnegativo que se encuentra en el medio acuático, en una gran variedad de hábitats diferentes. Hasta la fecha, se han identificado más de 49 especies, siendo la Legionella pneumophila la más habitual, y la causante de la enfermedad neumónica aguda denominada legionelosis, con predominio en pacientes inmunocomprometidos y fumadores ${ }^{1}$. Aunque los hallazgos radiográficos de esta entidad son variados y no específicos, las consolidaciones parcheadas periféricas en más de un lóbulo son la imagen inicial más habitual en la radiografía de tórax ${ }^{2,3}$. Las adenopatías reactivas hiliares o mediastínicas de tamaño significativo son un hallazgo excepcional en la neumonía por Legionella ${ }^{1,3}$. Aunque en la serie de Kim y colaboradores el TC torácico detectó adenopatías patológicas en un 40\% de los casos, en ninguno de estos pacientes las adenopatías eran visibles en la radiografía simple ${ }^{4}$. En otras series de neumonía por Legionella estudiadas por TC de tórax no se recoge la presencia adenopatías significativas en ningún caso $0^{5,7}$. En nuestro paciente la radiografía simple inicial mostró una masa parahiliar izquierda sin consolidación asociada, por lo que se sospechó un carcinoma pulmonar.

Figura 1. Masa que se proyecta sobre el hilio izquierdo con sutil aumento de densidad basal izquierdo

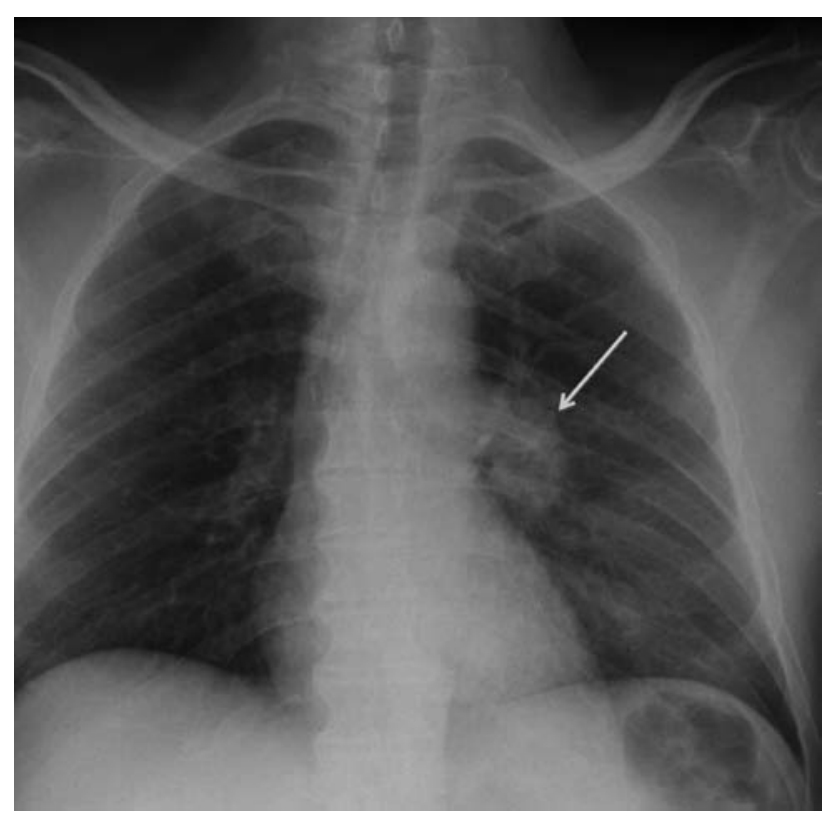


Figura 2. Extensas adenopatías:

Panel A paratraqueales izquierdas. Panel B hiliares y subcarinales izquierdas de tamaño significativo con lesión alveolar en todo el lóbulo inferior
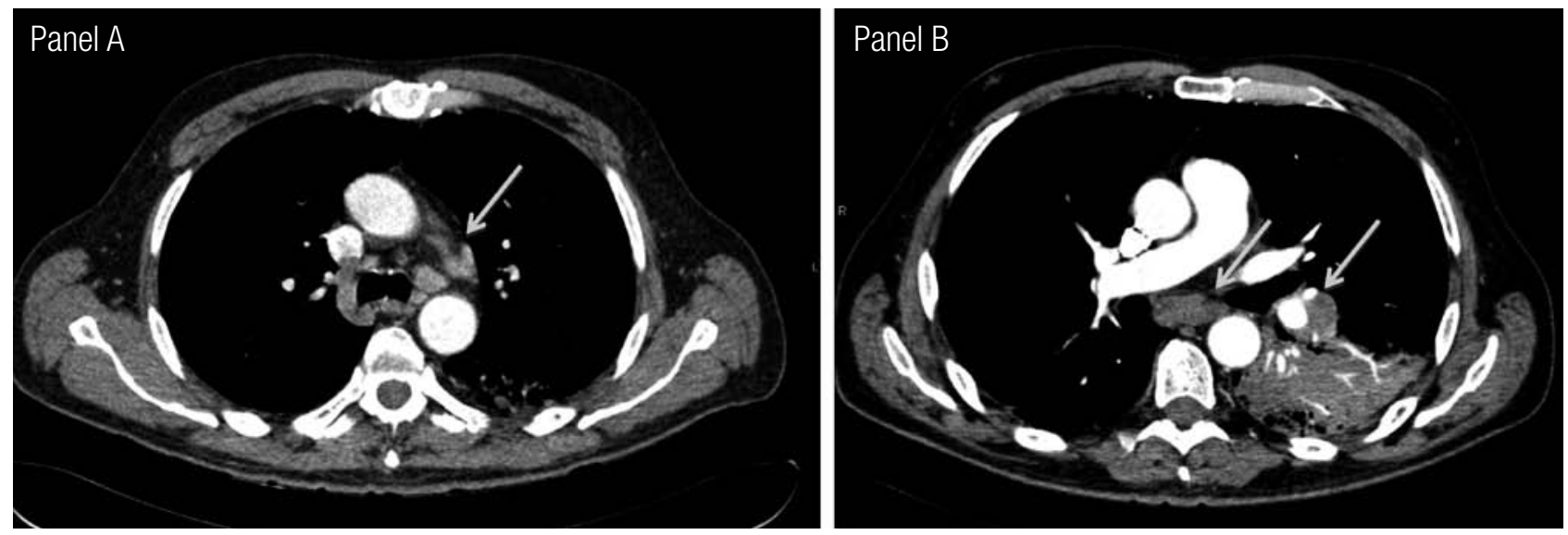

Es conocido que un $10 \%$ de las neumonías por Legionella pueden tardar hasta 72 horas en mostrar un infiltrado pulmonar identificable en la radiografía de tórax ${ }^{(2)}$, sin embargo, en la literatura revisada, no hemos encontrado ningún caso de masas adenopáticas, como forma de presentación de una neumonía por este patógeno. Durante la broncoscopia se realizó una punción transtraqueal de las adenopatías, que sólo mostró hiperplasia reactiva, documentándose resolución de las mismas en el estudio de TC torácico de control. Por todo ello y a pesar de los pocos datos existentes hasta la fecha, debemos tener presente que la neumonía por Legionella puede presentarse con grandes conglomerados de adenopatías, incluso simulando una masa pulmonar. Esto, unido a la clínica atípica de la que se puede acompañar, hace que esta entidad deba tenerse en cuenta en el diagnóstico diferencial de lesiones pulmonares sugestivas de neoplasia pero sin evidencia de malignidad en el estudio.

\section{Bibliografía}

1. Benito JR, Montejo JM, Cancelo L, Zalacain R, Lopez L, Fernandez Gil de Pareja J, et al. [Community-acquired pneumonia due to Legionella pneumophila serogroup 1. Study of 97 cases]. Enferm Infecc Microbiol Clin 2003; 21: 394-400.

2. Fairbank JT, Patel MM, Dietrich PA. Legionnaires' disease. J Thorac Imaging 1991; 6: 6-13.

3. Tan MJ, Tan JS, Hamor RH, File TM, Jr., Breiman RF. The radiologic manifestations of Legionnaire's disease. The Ohio Community-Based Pneumonia Incidence Study Group. Chest 2000; 117: 398-403.

4. Kim KW, Goo JM, Lee HJ, Lee HY, Park CM, Lee CH, et al. Chest computed tomographic findings and clinical features of legionella pneumonia. J Comput Assist Tomogr 2007; 31: 950-5.

5. Hui Y, Higa F, Hibiya K, Furugen M, Sato Y, Shinzato T, et al. Computed tomographic features of 23 sporadic cases with Legionella pneumophila pneumonia. Eur J Radiol 2010; 74: e73-8

6. Yagyu H, Nakamura H, Tsuchida F, Sudou A, Kishi K, Oh-ishi S, et al. Chest CT findings and clinical features in mild Legionella pneumonia. Intern Med 2003; 42: 477-82.

7. Sakai F, Tokuda H, Goto H, Tateda K, Johkoh T, Nakamura H, et al. Computed tomographic features of Legionella pneumophila pneumonia in 38 cases. J Comput Assist Tomogr 2007; 31: 125-31. 